\title{
Research on the Key Technology of Seismic Observation Equipment Clus- ter Monitoring
}

\author{
Wang Xiaoming ${ }^{*}, 1$, Du Lijuan ${ }^{2}$, Zhang Yong ${ }^{1}$, Zhao Xuezhi ${ }^{1}$, Tao Yinglu ${ }^{1}$ and Cheng Xianzhou ${ }^{1}$ \\ ${ }^{I}$ Earthquake Administration of Shanghai Municipality, Shanghai, 200062, P.R. China; ${ }^{2}$ School of air transportation, \\ Shanghai University of Engineering Science, Shanghai 201620, China
}

\begin{abstract}
This paper proposes a reactive monitoring system model, which is suitable for seismic observation equipment cluster. By analyzing two key factors affecting the performance of the system under our proposed model, we pose a priority queue based (PQB) polling mechanism as well as the volatility-removed (VR) fault alarm mechanism for the cluster node monitoring system. Our novel PQB polling mechanism initializes priorities of cluster nodes according to their importance and then puts cluster nodes into the polling queue according to their priority order. Nodes with same priority follow FCFS algorithm, thus solving the problems that important cluster nodes be monitored first. A formula judging whether cluster node is in the fluctuant state is given by VR fault alarm mechanism, which improves the accuracy of the monitoring results. Finally, we build an experimental platform to analyze the performance of PQB polling and VR warning mechanisms. Results show that PQB polling mechanism increases the number of polling nodes than that of FCFS polling mechanism; meanwhile, VR warning mechanism is more close to the actual fault results than normal alarm mechanism. Furthermore, PQB and VR mechanisms have no significant impact on server performance.
\end{abstract}

Keywords: Priority queue, Polling mechanism, Volatility removed, Fault alarm mechanism.

\section{INTRODUCTION}

Computer cluster contains interconnected and loose set of computers which can work together rapidly and closely. Cluster nodes are connected with LAN or Internet and have complete independent software and hardware [1]. Cluster is normally used to improve computing speed and reliability of one single computer. Through load balancing $[2,3]$ and parallel computing [4] technology, the cluster achieves higher efficiency, so it tends to be widely used in railway information system [5], command control [6], road and bridge tunnel health monitoring [7], etc. Because of its special targets and tasks, the seismic profession needs to deploy seismic monitoring nodes in various regions. With all kinds of data recorders, servers, route and switch devices either indoors or outdoors, a seismic monitoring distributed cluster network is contributed to ensure the seismic monitoring data be continuously and accurately transmitted to the center in real-time. Distributed cluster system has the characteristics of multiple fault nodes with different kinds of fault types, so there are potential faults and anomalies [8] in hardware, operating system, environment, internal network, cluster management and scheduling software, application and parallel computing environment, critical system services, storage file systems and so on. It is the same with such a huge earthquake monitoring equipments cluster since management and maintenance are arduous and important tasks for information department in seismic profession.

At present, the majority of monitoring systems of earthquake monitoring device cluster are based on an open source

\footnotetext{
*Address correspondence to this author at Earthquake Administration of Shanghai Municipality, Shanghai, 200062, P.R. China; Tel: +86 21 31759059; Fax:+86 21 31759070; E-mail: wangyoucao78@163.com
}

software such as Nagios, Cacti, etc. $[9,10]$. The central BUS system communicates with distributed software agents deployed in each node. Lots of practical problems will be encountered: Monitoring model selection error leads to low efficiency of monitoring system; Lack of detailed analysis of the relevant attribute of monitored nodes results in high load of central BUS system; Ignoring link status of monitored nodes causes inaccuracy of monitoring data; Imperfection of polling detection and alarm mechanism [11] leads to false or missing notifications of equipment fault information. In order to deal with the above problems, this paper mainly fulfills three tasks: Firstly, we propose a novel and practical cluster monitoring model for seismic profession through describing typical characteristics of nodes in seismic monitoring cluster. Secondly, we put forward the priority queue based (PQB) polling mechanism for the cluster node monitoring to cope with the ignorance of important nodes' monitoring problem. Thirdly, we pose the volatility-removed (VR) fault alarm mechanism to deal with the inaccuracy warnings for node fault information.

The rest of this paper is organized as follows. In Section 2 , related works on cluster monitoring system and model are presented. In Section 3, we describe the characteristics of nodes in seismic monitoring cluster and put forward the reactive model which is suitable for seismic monitoring cluster. Section 4 proposes the PQB polling mechanism as well as the VR fault alarm mechanism by formulating the volatility of nodes in cluster. Experimental results and evaluation are given in Section 5 followed by conclusions in Section 6 .

\section{RELATED WORKS}

Research efforts focusing on seismic monitoring devices cluster begin to increase in recent years due to frequent happening of earthquakes. The authors in [12] [13] use GPS 
technology to provide real-time monitoring for receiver system, AC and DC power system, network equipments, environment system in unattended stations. A wireless remote monitoring system based on $3 \mathrm{G}$ and SMS for seismic station is proposed in [14]. In [15], the remote network video monitoring on Karamay unattended station is carried out by using spread spectrum microwave communications with LAN connection. The authors in [16] deeply investigated open source software Nagios and built a seismic network monitoring system to achieve visual monitoring of devices and services in station. Also, a detailed introduction of principle, function and characteristic about Nagios software is proposed in [17]. The authors put forward the building process and configurations of monitoring system and show applications of seismic network monitoring in Heilongjiang. But there are problems in installation, configuration, automatic discovery and events controlling under this system. [18] points that the accuracy for monitoring results should be improved in Nagios based systems. Furthermore, authors in [19] discuss the analysis, processing and multi-level interactive distribution technology in handling warning information of seismic monitoring system. However, most of monitoring systems introduced above ignore the priorities of important nodes especially near seismic zones. Moreover, inaccuracy of fault alarms leads to unnecessary expenditures and efforts.

\section{SEISMIC DEVICES CLUSTER MONITORING MODEL}

In this section, we first describe the characteristics and link status of nodes in seismic monitoring cluster. Secondly, we propose monitoring model for seismic devices cluster.

\subsection{Characteristics and Link Status of Nodes in Seismic Monitoring Cluster}

Cluster nodes monitored are classified as station monitoring devices, application servers and router-switchers on the basis of functional properties in seismic profession. Among the nodes, seismic monitoring devices and router-switchers require only IP and port status monitoring while application servers should be installed with agents so as to monitor per- formance of operating systems such as CPU usage, memory usage, disk usage, etc. We must consider these various monitoring requirements in the cluster monitoring system deployment.

Seismic profession cluster nodes can be divided into SDH, CDMA (GSM, GPRS, 3G), satellite links according to communication link types. Different communication technologies are used due to the different types of transmission mediums and distances in different communication links, thus the requirements for nodes monitoring information in different links are different accordingly. Consequently, the diversity will influence on node polling and fault alarm mechanisms to be discussed in section 4. Taking seismic monitoring devices cluster in Earthquake Administration of Shanghai Municipality (EASM) as an example, link status and fault alarm thresholds for different types of communication links are set in Tables $\mathbf{1}$ and $\mathbf{2}$ respectively.

\subsection{Cluster Monitoring Model}

According to the node attributes and monitoring requirements, we propose a reactive monitoring model for seismic devices cluster monitoring system which agent is deployed in each cluster node.

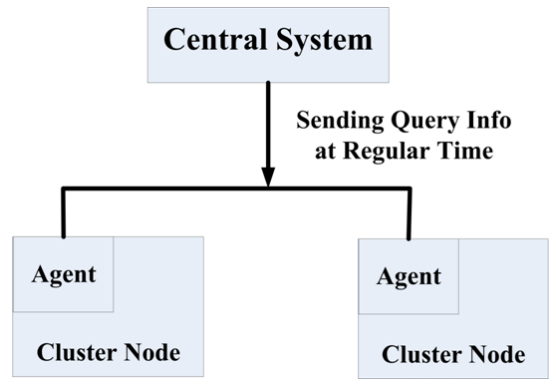

Fig. (1). Reactive monitoring model.

As shown in Fig. (1), the central system judges node status by the information sent by node passively, and then delivers feedbacks to users according to system alarm mechanism. In this model, central system can reasonably set polling and alarm mechanisms according to its performance,

Table 1. Alarm thresholds for different types of link status.

\begin{tabular}{|c|c|c|c|}
\hline $\begin{array}{l}\text { Devices States } \\
\text { Link Types }\end{array}$ & Normal & Warning & Critical \\
\hline $\mathrm{SDH}$ & $\begin{array}{c}\text { delay }<500 \mathrm{~ms} \\
\text { packet loss }<20 \%\end{array}$ & $\begin{array}{l}500 \mathrm{~ms} \leq \text { delay }<1000 \mathrm{~ms} \\
20 \% \leq \text { packet loss }<60 \%\end{array}$ & $\begin{array}{c}\text { delay } \geq 1000 \mathrm{~ms} \\
\text { packet loss } \geq 60 \%\end{array}$ \\
\hline Satellite & $\begin{array}{c}\text { delay }<1100 \mathrm{~ms} \\
\text { packet loss }<30 \%\end{array}$ & $\begin{array}{l}1100 \mathrm{~ms} \leq \text { delay }<2000 \mathrm{~ms} \\
30 \% \leq \text { packet loss }<60 \%\end{array}$ & $\begin{array}{c}\text { delay } \geq 2000 \mathrm{~ms} \\
\text { packet loss } \geq 60 \%\end{array}$ \\
\hline GPRS & $\begin{array}{c}\text { delay }<1000 \mathrm{~ms} \\
\text { packet loss }<30 \%\end{array}$ & $\begin{array}{l}1000 \mathrm{~ms} \leq \text { delay }<1800 \mathrm{~ms} \\
30 \% \leq \text { packet loss }<60 \%\end{array}$ & $\begin{array}{c}\text { delay } \geq 1800 \mathrm{~ms} \\
\text { packet loss } \geq 60 \%\end{array}$ \\
\hline ADSL & $\begin{array}{c}\text { delay }<500 \mathrm{~ms} \\
\text { packet loss }<20 \%\end{array}$ & $\begin{array}{l}500 \mathrm{~ms} \leq \text { delay }<1000 \mathrm{~ms} \\
20 \% \leq \text { packet loss }<60 \%\end{array}$ & $\begin{array}{c}\text { delay } \geq 1000 \mathrm{~ms} \\
\text { packet loss } \geq 60 \%\end{array}$ \\
\hline $3 \mathrm{G}$ & $\begin{array}{c}\text { delay }<800 \mathrm{~ms} \\
\text { packet loss }<30 \%\end{array}$ & $\begin{array}{l}800 \mathrm{~ms} \leq \text { delay }<1200 \mathrm{~ms} \\
30 \% \leq \text { packet loss }<60 \%\end{array}$ & $\begin{array}{c}\text { delay } \geq 1200 \mathrm{~ms} \\
\text { packet loss } \geq 60 \%\end{array}$ \\
\hline
\end{tabular}


Table 2. Alarm thresholds for different types of service fault.

\begin{tabular}{|c|c|c|c|}
\hline Service Status & Normal & Warning & Critical \\
\hline Windows & $\begin{array}{c}\text { CPU Usage }<80 \% \\
\text { Memory Usage }<80 \%\end{array}$ & $\begin{array}{c}80 \% \leq \text { CPU Usage }<90 \% \\
80 \% \leq \text { Memory Usage }<90 \%\end{array}$ & $\begin{array}{c}\text { CPU Usage } \geq 90 \% \\
\text { Memory Usage } \geq 90 \%\end{array}$ \\
\hline Linux (Including AIX) & $\begin{array}{c}\text { Current Load in } 1 \text { minute }<15 \\
\text { Current Load in } 5 \text { minutes }<10 \\
\text { Current Load in } 15 \text { minutes }<5 \\
\text { Current Users }<5 \\
\text { Total Processes }<150\end{array}$ & $\begin{array}{l}15 \leq \text { Current Load in } 1 \text { minute }< \\
30 \\
\begin{array}{r}10 \leq \text { Current Load in } 5 \text { minutes } \\
<25 \\
5 \leq \text { Current Load in } 15 \text { minutes } \\
<20 \\
5 \leq \text { Current Users }<10 \\
150 \leq \text { Total Processes }<200\end{array}\end{array}$ & $\begin{array}{c}\text { Current Load in } 1 \text { minute } \geq 30 \\
\text { Current Load in } 5 \text { minutes } \geq 25 \\
\text { Current Load in } 15 \text { minutes } \geq 20 \\
\text { Current Users } \geq 10 \\
\text { Total Processes } \geq 200\end{array}$ \\
\hline
\end{tabular}

thus improving system reliability and decreasing costs in communication links and system loads. The disadvantage of this model is that agent need to be deployed in each cluster node, which increases costs; furthermore, polling and alarm mechanisms of central system are highly required in reactive monitoring model. It must be considered how to achieve a compromised load balancing degree. Monitored nodes in seismic profession are servers, router-switchers, data recorders, UPS equipments, Geoelectrical Observation Apparatus, Vertical Pendulum Tiltmeters, Water Temperature Recorders and so on. Among all devices, most of them only need to be monitored on-off state through IP address except some servers, UPS devices and other additional equipments such as power controller [20], so polling and alarm mechanisms are to be concerned in minority of cluster nodes. This model is suitable for monitoring system in seismic profession.

\section{POLLING AND FAULT ALARM MECHANISMS}

\subsection{PQB Polling Mechanism}

The basic polling mechanism in cluster monitoring system is briefly described as follows: Central system collects required information from the cluster nodes according to the configurations. Nodes send status feedbacks to central system either directly or through agents. That kind of polling mechanism will lead to the following problems: First, important nodes will be ignored as all cluster nodes have equal priorities. In seismic profession, stationary (especially near the seismic zones) real-time observation devices and core databases are of utmost importance so they should be set with the highest priorities; Second, the ordinary cluster nodes status is normal over a long period of time. Based on classical trend analysis algorithms such as Linear Regression Algorithm and Exponential Regression Algorithm [21], such nodes' priorities should be lowered. To solve the above two problems, we propose the PQB polling mechanism for cluster nodes monitoring. Priorities of nodes are initialized in accordance with the importance degree in the cluster. Each node is put into the polling queue according to its priority. FCFS algorithm will be used when nodes' priorities are in equal. The priority minus one after the node is polled in case that the nodes cannot be polled over a long period of time because of their low priorities. Nodes are reinserted into polling queue until priorities are less than thresholds. Figs. (2a and $\mathbf{2 b}$ ) show the procedures of PQB polling mechanism.

\subsection{VR Alarm Mechanism}

Cluster monitoring system generates alarm information through fault alarm mechanism to inform user to deal with faults when node status reaches or exceeds the threshold initialized by configurations. Two aspects are included: alarm trigger and alarm coordination.

Most of the current network monitoring systems set fault alarm thresholds to trigger the fault alarm information. Seismic monitoring cluster nodes' tolerances to the network delays and congestions are different because of different types of the nodes, e.g. for seismic precursor devices, data acquisitions will be stored locally to avoid network delays and congestions, when the network returns to normal, stored results will be read from remote terminal. However, for seismic monitoring devices, data acquisitions should be sent to remote central systems in real-time, thus low delays and congestions of network are needed. In order to improve the reliability and robustness of the cluster monitoring system, we propose the VR fault alarm mechanism.

We identify four types of device status: normal, warning, critical, fluctuant. An interval threshold (TH) value is set between any other types of status to determine whether nodes are changed into fluctuant status in which node status changed frequently and irregularly. In this specific status, fault alarms should be prohibited so as to ensure the accuracy of alarms, reduce the load of central servers, and to prevent the generation of notification storms. We formulate the volatility as

$V_{n \bullet \Delta \mathrm{t}}=\frac{k}{n-1} \times 100 \%$

where $\Delta t$ is the polling cycle of monitoring system, $V_{n \bullet \triangle t}$ is volatility of node at time $n \bullet \Delta t$. The parameter $\mathrm{k}$ determines counts of node changes from time 0 to $n \bullet \Delta t$, and parameter $n-1$ determines expected counts of node changes from time 0 to $n \bullet \Delta t$. We assume that initial time $V_{0}=0$ and 


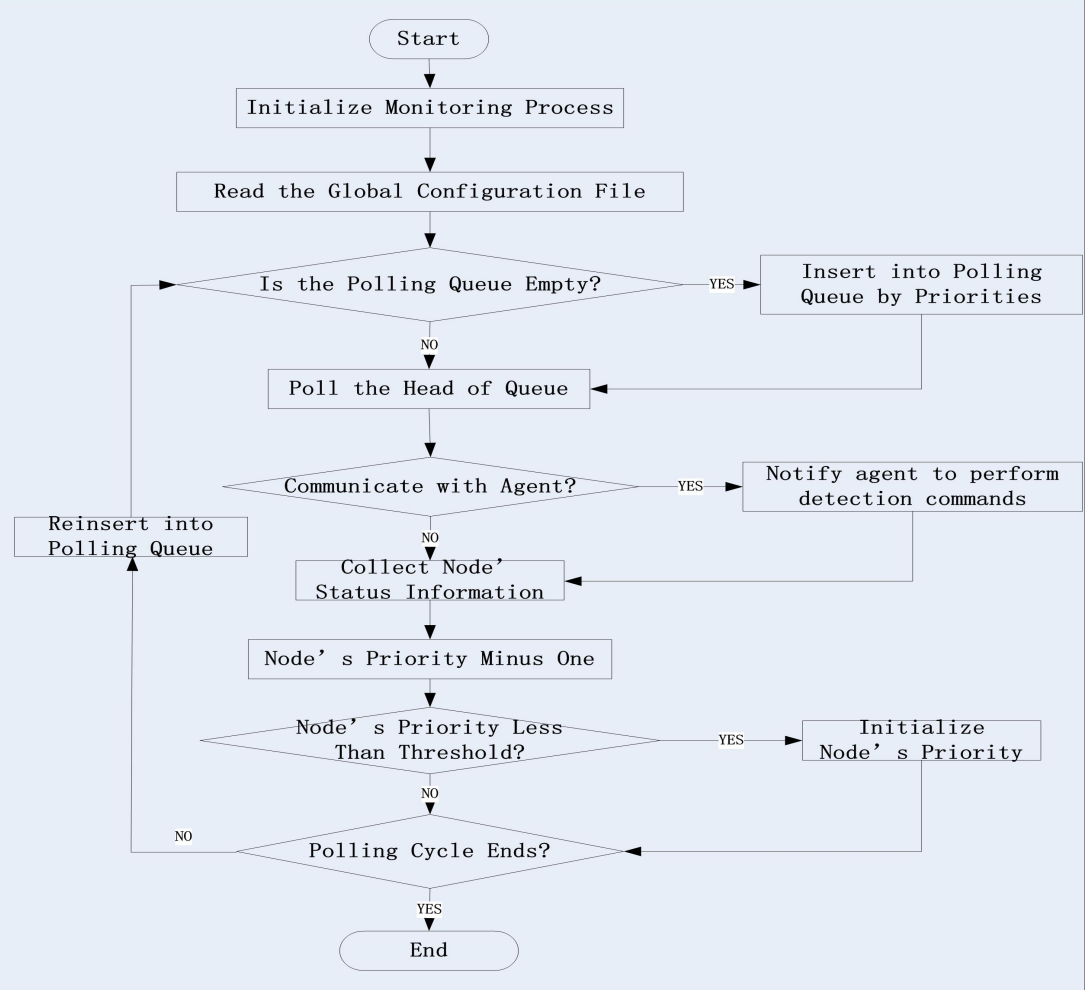

Fig. (2a). Central system monitoring flow chart.

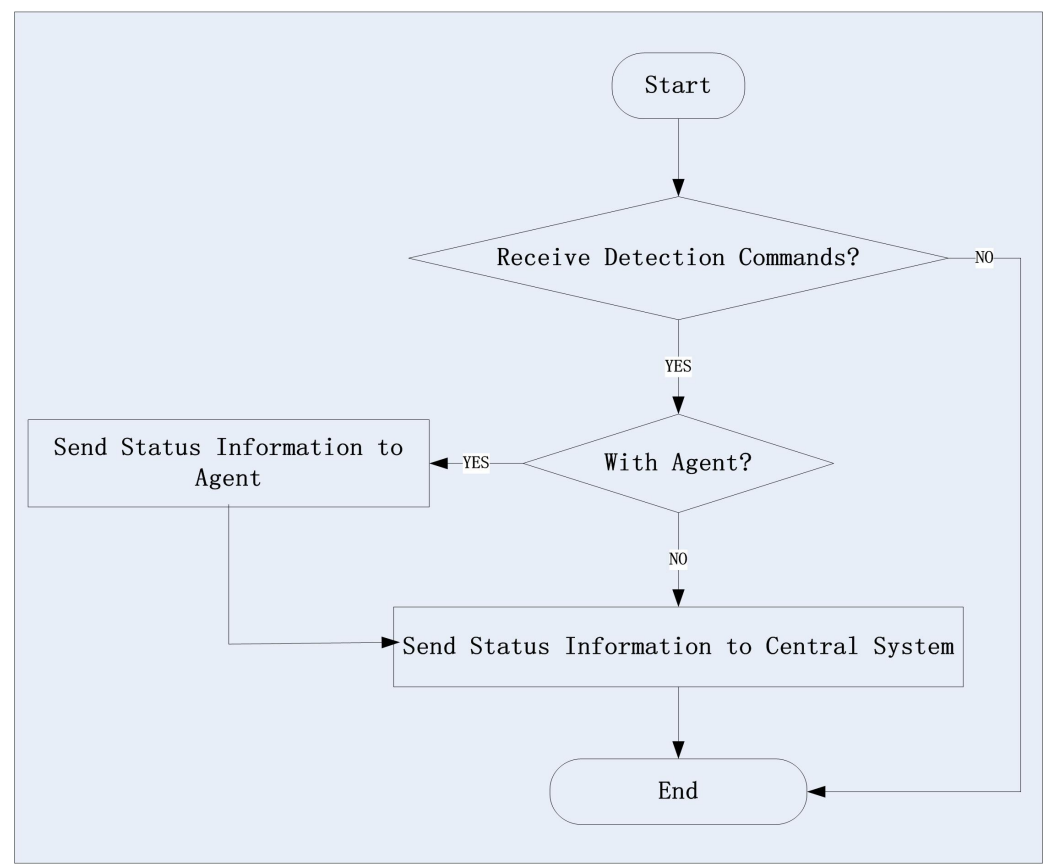

Fig. (2b). Cluster node information feedback flow chart.

initial node volatility is 0 . Fluctuation state at time $n \bullet \Delta t$ is calculated by formula

$$
F_{n \bullet \triangle \mathrm{t}}= \begin{cases}1, & \left(V_{n \bullet \triangle \mathrm{t}} \geq T H_{\text {max }}\right) \\ 0, & \left(V_{n \bullet \triangle \mathrm{t}} \leq T H_{\text {min }}\right)\end{cases}
$$

where $F_{n \bullet \triangle t}$ is the node fluctuation state determination at time $n \bullet \Delta t$, value 1 represents fluctuation state and value 0 represents the opposite. $T H_{\min }$ and $T H_{\max }$ represent the minimum and maximum of whether node is in or out of fluctuation state respectively. Faults notifications are forbidden when node is in the fluctuation state.

After determining on triggering the alarms, monitoring system need to notify users. What should be concerned are notification objects, notification commands and notification filtrations. Notification objects could be predefined and pre- 
Table 3. Experimental parameters.

\begin{tabular}{|c|c|c|}
\hline Parameters & Values & Descriptions \\
\hline CPU of Central Server & $\begin{array}{l}\text { Intel(R)Xeon }(\mathrm{R}) \\
5130 @ 2.00 \mathrm{GHz}\end{array}$ & \\
\hline Memory of Central Server & $4 \mathrm{G}$ & \\
\hline Length of Polling Queue & 248 & Total numbers of all station devices, central servers, routers and switchers, virtual machines \\
\hline $\begin{array}{c}\text { Numbers of Important } \\
\text { Nodes }\end{array}$ & 78 & Total numbers of core database, router and switcher, part of important station devices \\
\hline Polling Cycle t & 5 minutes & Time using for monitoring system polling once, set 5 minutes based on experience \\
\hline $\mathrm{TH}_{\min }$ & $5 \%$ & $\begin{array}{l}\text { Determination of the minimum threshold when node departs from fluctuating status, set } 5 \% \text { based } \\
\text { on experience }\end{array}$ \\
\hline $\mathrm{TH}_{\max }$ & $20 \%$ & $\begin{array}{c}\text { Determination of the maximum threshold when node gets into fluctuating status, set } 20 \% \text { based on } \\
\text { experience }\end{array}$ \\
\hline $\mathrm{n}$ & 30 & Counts of polling cycles during samples monitoring span, set 30 based on experience \\
\hline $\begin{array}{l}\text { Span of Experimental Data } \\
\text { Acquisition }\end{array}$ & 30 days & $\begin{array}{l}\text { In order to meet the accuracy requirements, we collected } 30 \text { days monitoring results which are from } \\
03 / 01 / 2014 \text { to } 03 / 31 / 2014 \text { (FCFS and normal alarm mechanisms)and from 04/01/2014 to } \\
\text { 05/01/2014(PQB and VR alarm mechanisms) }\end{array}$ \\
\hline
\end{tabular}

sorted in monitoring system for the purpose of delivering different notifications to different objects. By external extension to notification commands, notification methods could be web interface, voice, email, SMS, etc. For the sake of losing control of alarm messages, we should filter the information by determining to send cluster nodes' alarms or not, setting times for contacts to receive notifications, setting counts for alarm notifications, etc.

\section{EVALUATION}

We take central and stationary devices in EASM as samples and build an experimental platform based on Nagios [20], Cacti [21] monitoring systems to implement PQB polling mechanism and VR fault alarm mechanism. Parameters in the experiment are shown in Table 3. We make comparisons between two polling mechanisms about polling counts of important nodes as well as alarm notification counts between two fault alarm mechanisms according to 30 days' results respectively. Furthermore, comparisons about influences on central server performance are made.

It can be found in Fig. (3) that important nodes polling counts are approaching nearly linear over time under both FCFS and PQB polling mechanisms. Important nodes polling counts under PQB poling mechanism are higher than that of FCFS. This is because under this particular mechanism, all nodes are sequenced according to their priorities. Important nodes have higher priorities and will be polled by central system first, meanwhile, each polled node reinserts into polling queue according to some effective sort algorithms, which ensures the fairness of ordinary nodes. In addition, the threshold ensures priorities for nodes initialized with higher priorities in a long polling period. As a result, the PQB polling mechanism is suitable for seismic profession with great differences between cluster nodes.

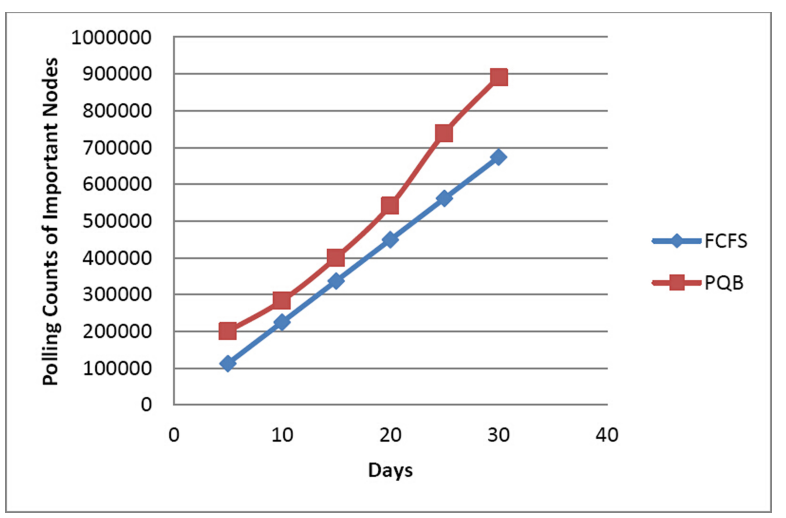

Fig. (3). Comparison of important nodes polling counts between two polling mechanisms.

Fig. (4) compares actual fault counts with that under ordinary fault alarm and VR fault alarm mechanisms respectively.

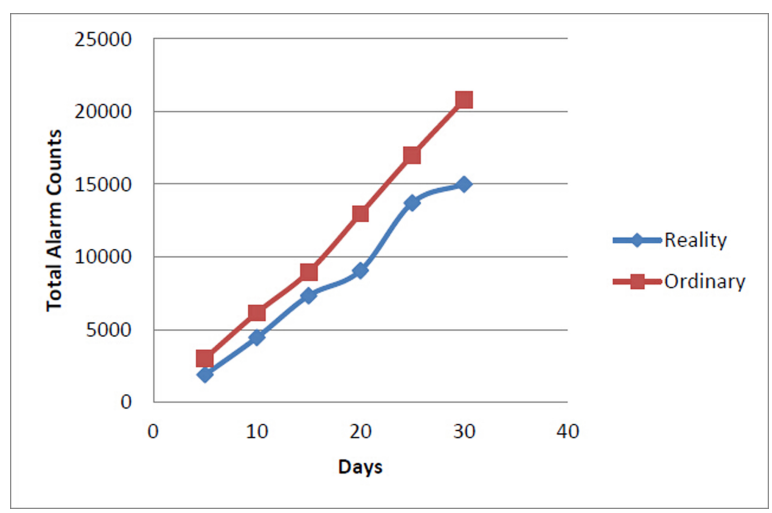

Fig. (4a). Counts comparison between normal alarm mechanism and actual fault. 


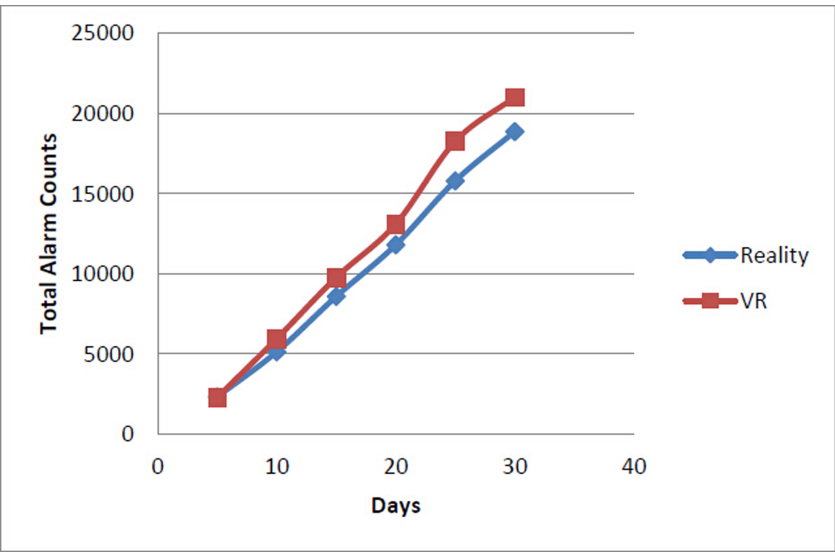

Fig. (4b). Counts comparison between VR alarm mechanism and actual fault.
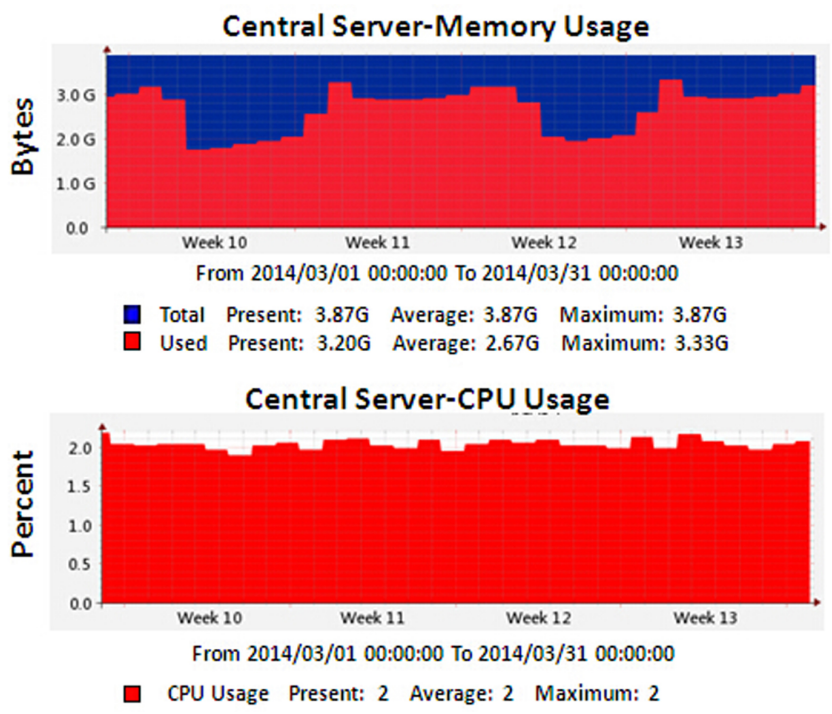

Fig. (5a). Effect of FCFS polling and normal alarm mechanisms on server performance.
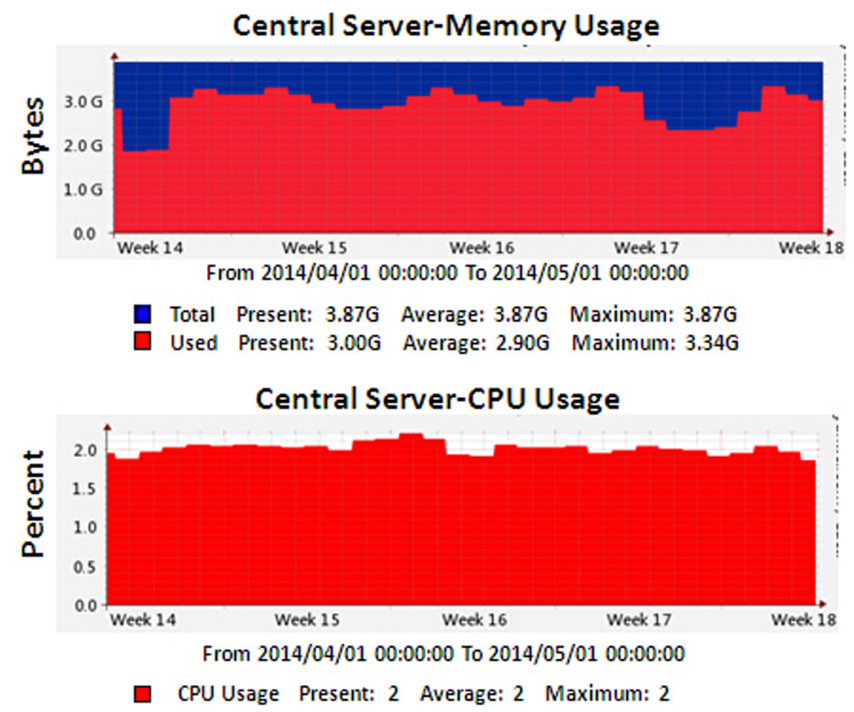

Fig. (5b). Effect of PQB polling and VR alarm mechanisms on server performance.
Fig. (4a) shows that alarm counts under ordinary fault alarm mechanism are higher than actual fault counts. That is because nodes' state changes rapidly under fluctuation status; moreover, each state change triggers an alarm message. However, nodes can tolerate network delays and congestions to some extent so that there would be a few false alerts in practice. Fig. (4b) displays that alarm counts under VR fault alarm mechanism gradually approach the actual values over time as this mechanism adds judgment to the fluctuation status and restricts frequent alerts under fluctuation status. Notably, not all alarm counts under VR mechanism are higher than that in practice, which is because various tolerance capacities among cluster devices lead to some false alerts. Obviously, VR fault alarm mechanism is close to reality compared with ordinary fault alarm mechanism.

Fig. (5) gives statistics of influence on server CPU and memory usages under FCFS, PQB polling mechanisms and ordinary, VR fault alarm mechanisms over 30 days respectively. From comparisons in Figs. (5a and 5b), we can draw the conclusion that the four polling and fault alarm mechanisms have little influence on the server CPU and memory usages. Within 30 days, the average server CPU usage under FCFS polling and ordinary alarm mechanisms is $2 \%$, while the result under PQB and VR mechanisms is $2 \%$, which means no significant effect on CPU usage. Within 30 days, the average server memory usage under FCFS polling and ordinary alarm mechanisms is $2.67 \mathrm{~GB}$, while the result under PQB and VR mechanisms is $2.90 \mathrm{~GB}$, which means little significant effect on memory usage. Both FCFS, PQB, ordinary, VR mechanisms can meet requirements for normal operation of the system, thus from what has been discussed above, new PQB and VR mechanisms will not cause excessive load of monitoring system.

\section{CONCLUSION}

This paper proposes a reactive monitoring model for cluster devices monitoring system according to the characteristics of seismic profession. Under this model, we study the shortcomings of the original FCFS polling mechanism and pose a PQB polling mechanism for supplement. New polling mechanism will solve the problem of great differences between cluster nodes by initializing priorities of cluster nodes in polling queue, and ensure the fairness of the cluster nodes by decreasing priorities of polled nodes and setting thresholds. Furthermore, we pose the VR fault alarm mechanism implemented by giving fluctuation status determination formula. The results from monitoring platform we built show that under PQB polling mechanism, polling counts of important nodes are increased compared with FCFS mechanism; meanwhile, VR fault alarm mechanism approaches more close to reality than ordinary fault alarm mechanism. More importantly, PQB and VR mechanisms have little significant influence on the performance of central server, so the mechanisms are suitable for seismic profession with great differences between cluster nodes.

\section{CONFLICT OF INTEREST}

The author confirms that this article content has no conflict of interest. 


\section{ACKNOWLEDGEMENTS}

This work was financially supported by Shanghai Science and Technology Committee program (15dz1207600) and China Scholarship Council.

\section{REFERENCES}

[1] Yang Yi. "Parallel Computing of Map Tile under PC Cluster", Science of Surveying and Mapping, Vol. 39, no. 3, pp. 120-123, 2014.

[2] ZHANG Chuang, GU Jian-hua. "A Load Balancing Strategy in Virtual Machine Cluster Based on Live Migration of Virtual Machine", Microelectronics and Computer, Vol. 31, no. 4, pp. 79-82, 2014.

[3] Wang Xiao-ming, Qin Hao-wen. "The conception and implementation of desktop virtualization technology in Earthquake Administration of Shanghai Municipality", Seismological and geomagnetic observation and research, Vol. 34, Issue 3/4, pp. 238-244, 2012.

[4] Aghila Rajagopal, M.A. Maluk Mohamed. "An Optimized Parallel Computing Paradigm for Mobile Grids Based on DSPOM", Journal of Emerging Technologies in Web Intelligence, Vol. 6, Issue 1, pp. 119-132, 2014.

[5] Roger G. Nyberg, Narendra Gupta, Siril Yella, Mark Dougherty. "Detecting Plants on Railway Embankments", Journal of Software Engineering and Applications, Vol. 6, Issue 3B, pp. 8-12, 2013.

[6] Dr.B. Ramamurthy, S. Bhargavi, Dr. R. Shashi Kumar. "Development of a Low-Cost GSM SMS-Based Humidity Remote Monitoring and Control system for Industrial Applications", International Journal of Advanced Computer Sciences and Applications, Vol.1, Issue 4, pp. 20-26, 2010.

[7] Huang Jia-qiang. "Research on Introduction of Public Security Wireless Communication System of Urban Rail Transit", Journal of Railway Engineering Society, Issue 11, pp.102-106, 2013.

[8] Zhang Yi, Chen Liang, Pang Jian. "Fault monitoring and management system for multiple computing clusters", Computer Engineering \& Science, Vol.35, Issue 11, pp. 54-61, 2013.

[9] Nagios Software (http://www.nagios.org).

[10] Cacti Software (http://www.cacti.net).

[11] Wang Xiao-ming. "The Integration and Application of Fetion Service in Seismic Software Systems", Technology for Earthquake Disaster Prevention, Vol.7, Issue 4, pp. 431-443, 2012.
[12] Zuo De-lin, Ding Wen-xiu, Peng Mao-lei. "Remote Monitoring System of Unattended GPS Station", Journal of Geodesy and Geodynamics, Vol.31, Issue 2, pp. 153-155, 2011.

[13] Xian-li Su, Xing-qun Zhan, Man-cang Niu, Yan-hua Zhang. "Receiver autonomous integrity monitoring availability and fault detection capability comparison between BeiDou and GPS", Journal of Shanghai Jiaotong University (Science), Vol. 19, Issue 3, pp. 313324, 2014.

[14] Sun Hong-zhi, Liu Yi-meng, Wang Xue-cheng, Lu Shan, Zhao Long-mei, Sun Kai-wei, Gao Ye-xin. "The Realization of the Seismic Station's Wireless Remote Monitoring and 3G Backup Channel Scheme", Journal of Disaster Prevention and Reduction, Vol.30, Issue 1, pp. 45-49, 2014

[15] Sheng Yan, Liu Fu-an, Bi Hui-juan, Liu Pan. "Implementation of Remote Network Monitoring System in KAREMARY Unattended Seismic Station", Inland Earthquake, Vol. 26, Issue 3, pp. 286-290, 2012.

[16] Temuqile, Song Hua, LIU Ke, Zhang Yi-mei, Li Yin. "Application of Nagios in Earthquake Monitoring Network", Journal of Yangtze River Scientific Research Institute, Vol.28, Issue 11, pp. 36-41, 2011.

[17] Gao Dong-hui, Meng Xiang-long, Zhang Shou-guo, Yan Feng, Qiu Feng-ping, Gao Hong-wei. "Application of Network Monitoring System Based on Nagios in Heilongjiang Seismic Monitoring Network", Journal of Disaster Prevention and Reduction, Vol.29, Issue 2, pp. 67-73, 2013

[18] Wu Xiao-yan, Zhou Hai-sheng, Ji Xiang. "Discussion and Application of Nagios in Seismic Network Management", Plateau Earthquake Research, Vol.22, Issue 3, pp. 58-60, 2010.

[19] Li Gang, Wang Xiao-lei, Sun Lu-qiang, Yao Lan-yu, Zhou Li-xia, Qi Shi-chao, Yao Hui-qin, Liu Wen-bing. "Application of the Integrated Short Message Warning System Based on Nagios Software to the Earthquake Profession", Journal of Seismological Research, Vol.35, Issue 1, pp. 133-138, 2012.

[20] Wang Xiao-ming, Chen Jing, Zhang Yong. "The implementation of Remote Management Unit of State Power", Northwestern Seismological Journal, Vol. 35, Issue Suppl, pp. 158-161, 2013.

[21] Liang Ying-hong, Liu Yi-chun. "Trend analysis method for monitoring metrics in monitor system", Computer Engineering and Applications, Vol.49, Issue 12, pp. 218-222, 2013.

Received: May 16, 2015

Revised: August 23, 2015

Accepted: September 31, 2015

(C) Liu and Qiu; Licensee Bentham Open

This is an open access article licensed under the terms of the (https://creativecommons.org/licenses/by/4.0/legalcode), which permits unrestricted, non-commercial use, distribution and reproduction in any medium, provided the work is properly cited. 